\title{
EFEITOS DE DIFERENTES CONDIÇÕES DE IRRIGAÇÃO E DENSIDADE DE SEMEADURA NO MICROCLIMA E NA OCORRÊNCIA DE HELMINTOSPORIOSE E DE OÍDIO EM TRIGO $\left(^{1}\right)$
}

\author{
PAULO CESAR SENTELHAS $\left({ }^{2}\right)$, MÁRIO JOSE PEDRO JÚNIOR $\left({ }^{2,4}\right)$ \\ e JOÃO CARLOS FELÍCIO ( ${ }^{3}$ )
}

\begin{abstract}
RESUMO
Avaliou-se, em Campinas (SP), o efeito da irrigação por aspersão e da densidade de semeadura na temperatura média do ar e na duração do periodo de molhamento (DPM), e sua influência em helmintosporiose e oidio na cultura do trigo, em experimentos ao nível de campo durante os invernos de 1990 e 1991 respectivamente. Os resultados foram submetidos a análises estatísticas: de diferença de dados pareados (microclima) e de intervalos de confiança (taxa de crescimento das moléstias). As áreas com maior densidade de plantas e irrigadas mostraram a seguinte tendência: as temperaturas médias foram mais baixas, $0,7^{\circ} \mathrm{C}$, e a DPM, com maior duração, 45 minutos. Com relação às taxas de crescimento, notou-se que foram influenciadas positivamente pela irrigação e pela densidade de semeadura quando as condiçðes macroclimáticas não eram amplamente favoráveis ao desenvolvimento dos patógenos.
\end{abstract}

Termos de inderação: trigo, irrigação, densidade de semeadura, microclima e moléstias.

\section{ABSTRACT \\ EFFECTS OF DIFFERENT CONDITIONS OF IRRIGATION AND CROP DENSITY IN MICROCLIMATE AND SPOT BLOTCH AND POWDERY MILDEW OCCURRENCE}

Field experiments (1990 and 1991) were carried out in Campinas, State of São Paulo, Brazil, during winter season, in order to study sprinkler irrigation and crop density effects on micrometeorological parameters (average air temperature and dew duration) and to evaluate their influence in the occurrence of spot blotch and powdery mildew in wheat crop. The results obtained were submitted to statistical analysis: comparison of means of two independent sample analysis (microclimate) and confidence interval analysis (infection rates). It was verified that in the most dense and irrigated plots the average air temperatures were lower $\left(0.7^{\circ} \mathrm{C}\right)$ and the

(1) Parte da dissertação do primeiro autor como requisito para a obtenção do título de Mestre em Agronomia (Agrometeorologia). Recebido para publicaçāo em 23 de outubro de 1992 e aceito em 30 de abril de 1993.

$\left(^{2}\right)$ Seção de Climatologia Agrícola, Instituto Agronômico (IAC). Caixa Postal 28, $13001-970$ Campinas (SP).

$\left({ }^{3}\right)$ Seção de Arroz e Cereais de Inverno, IAC.

$\left.{ }^{4}\right)$ Bolsista do CNPq. 
dew durations (DPM) were longer (45 minutes) than in the other plots. The infection rates of diseases were influenced positively by irrigation and crop density when the macroclimatic conditions were not favorable to disease development.

Inder terms: wheat, irrigation, crop density, microclimate and diseases.

\section{INTRODUÇÃO}

A utilização da irrigação nas regiões produtoras de trigo irrigado tem sido cada vez mais freqüente, visto que os índices pluviométricos durante o inverno, no Estado de São Paulo, são geralmente insuficientes para $o$ atendimento da demanda hídrica da cultura.

A irrigação, principalmente a realizada por aspersão, normalmente induz alterações microclimáticas na cultura, em especial na temperatura, na umidade e na duração do ar e do período de molhamento (DPM). Esses parâmetros, fundamentais nos processos epidemiológicos, são afetados pelo adensamento da cultura (Blad et al., 1978; Sutton et al., 1984; Pedro Jr., 1989).

Segundo Barros (1988), um dos grandes problemas enfrentados pelos produtores de trigo são as doenças que ocorrem na cultura, sobretudo quando as condições meteorológicas são altamente favoráveis. Felício et al. $(1986,1988)$ verificaram que as moléstias do trigo têm ocorrido com elevada freqüência $e$ intensidade em anos extremamente chuvosos ou onde a irrigação por aspersão tem sido empregada, fazendo com que sejam intensificados os controles químicos, concorrendo para o aumento do custo de produção.

No caso da irrigação, isso acontece, segundo Rotem \& Palti (1969), porque a água aplicada por aspersão, além de provocar alterações no microclima, supre a umidade requerida para a infecção (germinação e penetração) de numerosos patógenos, favorecendo também a dispersão dos esporos, através dos respingos para outras plantas (Menzies, 1967).

O objetivo deste trabalho foi verificar o efeito da irrigação por aspersão e da densidade de semeadura no microclima da cultura do trigo e na epidemiologia da helmintosporiose (Helminthosporium sativum) e do oídio (Erysiphe graminis).

\section{MATERIAL E MÉTODOS}

$O$ experimento foi desenvolvido sob condiç̃̃es de campo, no Centro Experimental de Campinas do Instituto Agronômico (lat. $=22^{\circ} 55$ ' $\mathrm{S}$, long. $=$ $47^{\circ} 05^{\prime} \mathrm{W}$ e alt. $=669 \mathrm{~m}$ ), em duas fases: a primeira, realizada em $24 / 4$ a $6 / 9$ de 1990 e a segunda, em $4 / 6$ a $11 / 10$ de 1991 . O cultivar de trigo utilizado foi o IAC 24 e 0 espaçamento entre linhas, de $17 \mathrm{~cm}$.

Os tratamentos, em número de quatro, foram esquematizados, combinando-se duas condições de irrigação e duas densidades de semeadura (Quadro 1). Na primeira fase, 1990 , as subáreas de cada tratamento eram de $20 \times 20 \mathrm{~m}$ e na segunda, 1991, de $20 \times 10 \mathrm{~m}$, demarcadas dentro de uma área total de 2 ha e separadas entre si por uma distância de $30 \mathrm{~m}$.

A densidade de plantas por hectare nos dois anos de experimentação diferiram devido a problemas na porcentagem de germinação.

As irrigaçðes foram diferenciadas da seguinte forma: sequeiro (SI), com irrigaçôes somente no início do desenvolvimento da cultura para garantir seu estabelecimento, e irrigada (CI), com a frequêencia monitorada pelo método climatológico (Alfonsi et al., 1990).

No centro de cada subárea (tratamentos), foram instalados, ao nível do terço superior da cultura, os equipamentos meteorológicos: termoigrógrafo, para registro da temperatura e umidade relativa (dentro de um microabrigo termométrico), e aspergígrafo, para registro da duração do periodo de molhamento (DPM). A escolha do aspergigrafo baseou-se em testes realizados por Amador (1987) e Sentelhas (1992), nos quais o aparelho apresentou boas respostas.

Para avaliação da severidade das doenças (helmintosporiose e oídio), foram marcadas vinte plantas por tratamento, baseando-se as leituras 
realizadas nas escalas de James (1971), com freqüência semanal, desde os primeiros estádios fenológicos. A ocorrência das doenças avaliadas deu-se da seguinte forma: helmintosporiose, em 1990, com níveis insignificantes de oídio; e oídio, em 1991, sem a manifestação de helmintosporiose. Isso se deveu, provavelmente, à grande diferença climática ocorrida nos dois anos (Quadro 2).

Posteriormente, os dados obtidos de severidade da helmintosporiose (1990) e do oídio (1991) foram linearizados pelo modelo monomolecular, para determinação das taxas de crescimento. Esse modelo foi o que melhor ajustou os dados em teste realizado previamente, segundo o método de Campbell \& Madden (1990).

Para determinação da influência da irrigação e da densidade de plantio no microclima da cultura, os dados de temperatura média do ar e da DPM foram agrupados em períodos de cinco dias (médias qüinqüidiais) e comparados entre os diferentes tratamentos mediante "análise da diferença de dados pareados" (Sokal \& Rohlf, 1969) para os dois anos. Para a determinação da influência nas doenças, as taxas de crescimento médias de cada tratamento foram comparadas entre si pela "análise de intervalos de confiança" (Snedecor \& Cochran, 1973).

\section{RESULTADOS E DISCUSSÃo}

\subsection{Influência nas condiç̋̄es microclimáticas}

O quadro 3 apresenta os resultados da análise da diferença de dados pareados para temperatura do ar e DPM na cultura do trigo, indicando ter havido influência, em ambos os anos, da irrigação e da densidade nas temperaturas dentro do dossel. Os tratamentos mais densos e irrigados mostraram tendência de temperaturas mais amenas $\left(0,7^{\circ} \mathrm{C}\right)$ que os menos densos e não irrigados, concordando com os resultados de Rotem \& Palti (1969) e Blad et al. (1978). A única exceção foi observada nos tratamentos irrigados durante o segundo ano do experimento (1991), onde as temperaturas não diferiram estatisticamente entre os tratamentos mais e menos adensados. Isso pode ser explicado pelo efeito termorregulador da água, sempre presente nessas áreas devido às freqüentes irrigaçðes necessárias durante 0 ciclo da cultura (Quadro 2).

Quadro 1. Características dos tratamentos utilizados nos anos de experimentação (1990-91) com a cultura do trigo

Densidade

n:

milhðes plantas/ha

1990

$\begin{array}{lcc}\text { SI-D } & 4 & 1,3 \\ \text { CI-D } & 6 & 1,3 \\ \text { SI+D } & 4 & 1,7 \\ \text { CI+D } & 6 & 1,7 \\ & 1991 & \\ \text { SI-D } & 3 & 1,5 \\ \text { CI-D } & 10 & 1,5 \\ \text { SI+D } & 3 & 2,6 \\ \text { CI+D } & 10 & 2,6\end{array}$

SI-D: sem irrigação e menor densidade; CI-D: com irrigaçăo e menor densidade; SI+D: sem irrigação e maior densidade; C+D: com irrigaçăo e maior densidade. 
QUADRO 2. Condições termopluviométricas normais, média de 30 anos, e observadas, número de irrigaçð̃es efetivas, total de precipitação e irrigação e evapotranspiração máxima, durante os períodos do experimento na cultura do trigo

\begin{tabular}{|c|c|c|c|c|c|c|c|}
\hline \multirow{2}{*}{ Período } & \multicolumn{2}{|c|}{ Normais } & \multicolumn{2}{|c|}{ Observadas } & \multirow{2}{*}{$\begin{array}{l}\text { Irrigações } \\
\text { efetivas }\end{array}$} & \multirow{2}{*}{$\begin{array}{c}\text { Total } \\
\mathrm{P}+\mathrm{I}\end{array}$} & \multirow{2}{*}{$\begin{array}{c}\text { ETM } \\
\text { EST }\end{array}$} \\
\hline & TMED & PREC & TMED & PREC & & & \\
\hline & ${ }^{\circ} \mathrm{C}$ & $\mathbf{m m}$ & ${ }^{\circ} \mathrm{C}$ & $\mathrm{mm}$ & $\mathrm{n}^{\circ}$ & $\mathbf{m m}$ & $\mathrm{mm}$ \\
\hline 1990 & 19,0 & 37 & 17,5 & 94 & 2 & 214 & 213 \\
\hline 1991 & 19,8 & 40 & 19,3 & 3 & 7 & 303 & 250 \\
\hline
\end{tabular}

TMED: Temperatura média do ar, PREC: total de precipitação pluvial; P + I: total de precipitação mais irrigaçăo; ETM EST: evapotranspiração máxima estimada; Irrigaçðes efetivas - número total de irrigaçð̋es menos as realizadas para estabelecimento da cultura.

QUADRO 3. Análise da diferença de dados pareados de temperatura e đuração do periodo do molhamento dentro da cultura do trigo, nos dois anos de experimentação para diferenciação das condições microclimáticas

\begin{tabular}{llll} 
Ano & Tratamentos & Diferença \\
comparados & observada & $\begin{array}{c}\text { Resultado } \\
\text { resumido }\end{array}$ \\
\hline
\end{tabular}

1991

$S I-D \times S I+D$
$C I-D \times C I+D$
$S I+D \times C I+D$
$S I-D \times C I-D$

Temperatura $\left({ }^{\circ} \mathrm{C}\right)$

1990

SI-D $\times$ SI+D

CI-D $\times \mathrm{CI}+\mathrm{D}$

$\mathrm{SI}+\mathrm{D} \times \mathrm{CI}+\mathrm{D}$

$0,7 * *$

$0,7^{* *}$

$-\mathrm{D}>+\mathrm{D}$

$1,3 * *$

$-\mathrm{D}>+\mathrm{D}$

$1,3 * *$

SI $>$ CI

SI-D x CI-D

$0,3 * *$

SI $>$ CI

$0,0 \mathrm{~ns}$

$-\mathrm{D}>+\mathrm{D}$

$0,7^{* *}$

$-\mathrm{D}=+\mathrm{D}$

$0,9 * *$

SI $>$ CI

SI $>$ CI

\section{DPM (horas)}

1990

$$
\begin{aligned}
& S I-D \times S I+D \\
& C I-D \times C I+D \\
& S I+D \times C I+D \\
& S I-D \times C I-D \\
& \text { SI-D } \times S I+D \\
& C I-D \times C I+D \\
& \text { SI+D } \times C I+D \\
& \text { SI-D } \times C I-D
\end{aligned}
$$

1991

$$
\begin{aligned}
& 0,7^{*} \\
& 0,1 \mathrm{~ns} \\
& 0,1 \mathrm{~ns} \\
& 0,6 \mathrm{~ns} \\
& 0,5^{* *} \\
& 1,3^{* *} \\
& 1,5^{*} \\
& 0,6^{*}
\end{aligned}
$$$$
-\mathrm{D}<+\mathrm{D}
$$$$
-D=+D
$$$$
\mathrm{SI}=\mathrm{CI}
$$$$
\mathrm{SI}=\mathrm{CI}
$$$$
\text { -D }<+ \text { D }
$$$$
-D=+D
$$$$
\text { SI }<\text { CI }
$$$$
\mathrm{SI}<\mathrm{CI}
$$

ns - năo significativo estatisticamente. *: significativo ao nível de $5 \%$. **: significativo ao nível de $1 \%$.

SI-D: sem irrigaçăo e menos denso; CI-D: com imigação e menos denso; SI+D: sem irrigação e mais denso; CI+D: com irrigaçăo e mais denso. 
Com relação à DPM, os resultados são bastante distintos. Em 1990, somente houve diferença significativa entre os tratamentos não irrigados com diferentes densidades. Os demais não apresentaram diferenças estatísticas na DPM, provavelmente por esse ano ter sido chuvoso, durante o período estudado, exigindo poucas irrigações suplementares (Quadro 2), fazendo com que a presença de água na cultura fosse freqūente em todos os tratamentos.

Entretanto, em 1991, as áreas mais densas e irrigadas foram sempre mais úmidas, ou seja, com a DPM maior, mostrando a influência mais acentuada dessas duas práticas sob condições climáticas extremas, onde a irrigação, segundo Rotem \& Palti (1969), além de afetar a temperatura e a umidade, promove também maior desenvolvimento da vegetação: a mais densa irá influenciar na velocidade da evaporação da água proveniente da irrigação.

\subsection{Influência na taxa de crescimento das doenças}

As análises de intervalos de confiança, para verificação da influência da irrigação e da densidade de plantio nas taxas de crescimento da helmintosporiose e do oídio, encontram-se, respectivamente, nos quadros 4 e 5 .

QUADRO 4. Análise estatística de intervalos de confiança da taxa de crescimento da helmintosporiose na cultura do trigo, sob diferentes condiçðes microclimáticas (1990)

\section{Amostragens}

Taxa de Crescimento (/Unidade/Dia)

\begin{tabular}{rllll} 
& SI-D & CI-D & SI+D & CI+D \\
\hline 1 & 0,060 & 0,058 & 0,029 & 0,043 \\
2 & 0,071 & 0,066 & 0,046 & 0,026 \\
3 & 0,153 & 0,066 & 0,069 & 0,036 \\
4 & 0,114 & 0,028 & 0,033 & 0,055 \\
5 & 0,037 & 0,089 & 0,022 & 0,024 \\
6 & 0,063 & 0,035 & 0,078 & 0,107 \\
7 & 0,098 & 0,061 & 0,083 \\
8 & 0,044 & 0,079 & 0,113 & 0,045 \\
9 & 0,047 & 0,061 & 0,076 \\
10 & 0,061 & 0,075 & 0,057 & 0,030 \\
11 & 0,092 & 0,092 & 0,100 & 0,037 \\
12 & 0,066 & 0,049 & 0,056 & 0,052 \\
13 & 0,086 & 0,043 & 0,022 & 0,035 \\
14 & 0,070 & 0,066 & 0,077 & 0,098 \\
15 & 0,053 & 0,115 & 0,076 & 0,076 \\
16 & 0,036 & 0,060 & 0,043 & 0,058 \\
17 & 0,042 & 0,094 & 0,026 & 0,049 \\
18 & 0,055 & 0,061 & 0,074 & 0,039 \\
19 & 0,058 & 0,045 & 0,033 & 0,098 \\
20 & 0,028 & 0,033 & 0,109 & 0,147 \\
d & 0,014 & 0,011 & 0,014 & 0,015 \\
Média & $0,067 a$ & $0,064 a$ & 0,014 & $0,061 a$
\end{tabular}

SI-D: sem irrigação e menos denso; CI-D: com irrigação e menos denso; SI+D: sem irrigação e mais denso; CI+D: com irrigação e mais denso: $d$ : intervalo de confiança. Todas as médias seguidas de letras iguais não apresentam diferença estatística. 
Os resultados permitem verificar que a helmintosporiose (Figura 1A) não apresentou diferença entre as taxas de crescimento nos quatro tratamentos, o que se explica por terem sido, no periodo do experimento, as condições macroclimáticas amplamente favoráveis ao desenvolvimento do fungo, não tendo as alterações microclimáticas exercido nenhuma influência no crescimento do patógeno. Isso é bem explicado por Rotem et al. (1970), os quais concluíram que, sob condiçðes climáticas altamente favoráveis ou desfavoráveis à doença, nem a densidade da cultura nem a frequêencia da irrigação têm efeito sobre o desenvolvimento dos patógenos. O mesmo não ocorre sob condiçōes marginais, onde essas práticas são fundamentais para 0 estabelecimento do processo infeccioso.

Por outro lado, as taxas de crescimento do oídio, em 1991 (Figura 1B) foram bastante influenciadas pela densidade da cultura e pela irrigação, principalmente por esse ano ter sido menos favorável às doenças, com poucas chuvas (Quadro 2), tendo as alteraçóes microclimáticas

QUADRO 5. Análise estatística de intervalos de confiança da taxa de crescimento do oídio na cultura do trigo, sob diferentes condições microclimáticas (1991)

\begin{tabular}{rlllc} 
Amostragens & SI-D & CI-D & SI+D & CI+D \\
\hline 1 & 0,017 & 0,034 & 0,112 & 0,010 \\
2 & 0,017 & 0,017 & 0,026 & 0,018 \\
3 & 0,020 & 0,031 & 0,035 & $\ldots$ \\
4 & 0,029 & 0,016 & 0,040 & 0,023 \\
5 & 0,070 & 0,029 & 0,069 & 0,015 \\
6 & 0,018 & 0,032 & 0,043 & 0,005 \\
7 & 0,006 & 0,031 & 0,036 & 0,021 \\
8 & 0,019 & 0,023 & $\ldots$ & 0,030 \\
9 & 0,020 & 0,032 & 0,017 & 0,026 \\
10 & 0,011 & 0,045 & 0,056 & 0,013 \\
11 & 0,037 & 0,019 & 0,026 & 0,037 \\
12 & 0,006 & 0,032 & 0,039 & 0,010 \\
13 & 0,011 & 0,012 & 0,010 & 0,021 \\
14 & 0,008 & 0,016 & 0,057 & $\ldots$ \\
15 & 0,036 & 0,014 & 0,025 & 0,022 \\
16 & 0,008 & 0,018 & 0,023 & 0,026 \\
17 & 0,009 & 0,027 & 0,017 & 0,027 \\
18 & 0,004 & 0,078 & 0,040 & 0,027 \\
19 & 0,017 & 0,005 & 0,027 & 0,020 \\
20 & 0,024 & 0,026 & 0,036 & 0,005 \\
d & 0,0045 & 0,0072 & 0,0112 & 0,0044 \\
Média & $0,0162 b$ & $0,0269 \mathrm{ab}$ & $0,0386 \mathrm{a}$ & $0198 \mathrm{~b}$ \\
\hline
\end{tabular}

Taxa de Crescimento (/Unidade/Dia)

SI-D: sem irrigaçăo e menos denso; CI-D: com irrigação e menos denso; SI+D: sem irrigação e mais denso; CI+D: com irrigaçăo e mais denso; d: intervalo de confiança.

Todas as médias seguidas de letras iguais não apresentam diferença estatística. 
modificado o progresso do oídio. Nesse caso, a irrigação e a densidade de semeadura apresentaram uma influência progressiva nas taxas de crescimento, ou seja, do tratamento sem irrigação e menos denso para o irrigado e mais denso, confirmando as constatações de Barros (1988), de que em áreas irrigadas o potencial de danos de oídio é elevado. Portanto, pôde-se verificar que tanto a irrigação por aspersão como a densidade de semeadura provocaram alteraçðes no microclima e influenciaram o desenvolvimento de helmintosporiose e de oídio do trigo, dependendo, porém, das condições macroclimáticas vigentes.
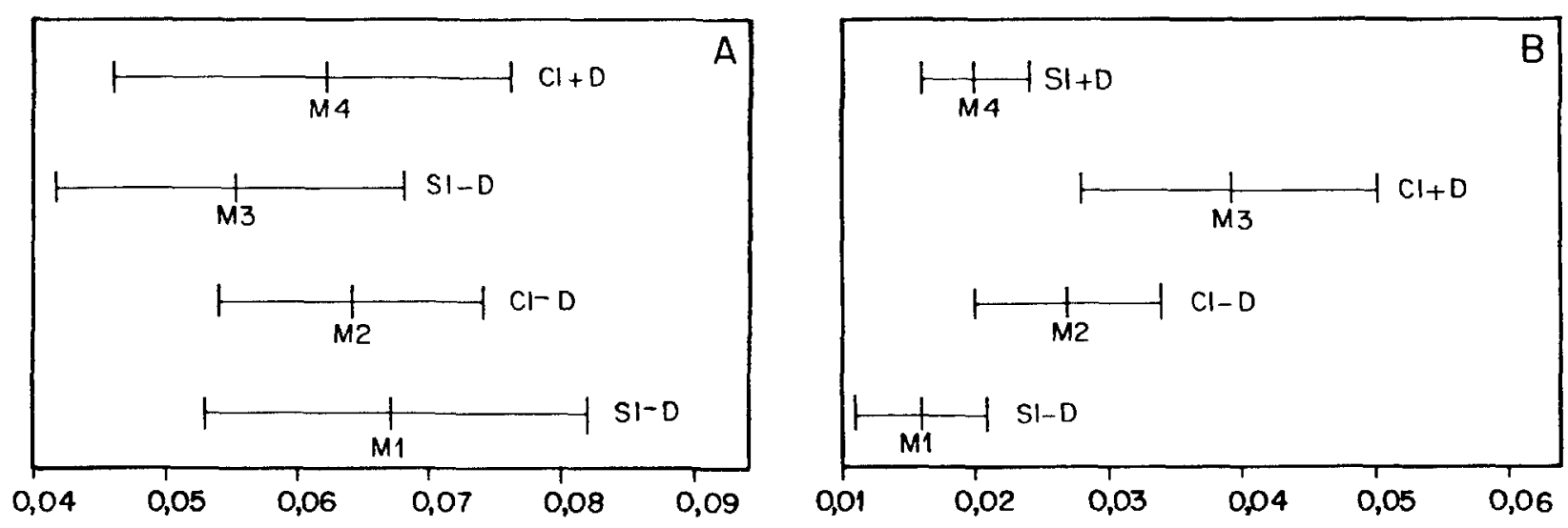

TAXA DE CRESCIMENTO MÉDIA, /Unidade/dia

Figura 1. Intervalos de confiança entre as taxas de crescimento médias. A: da helmintosporiose (1990) e B: do oídio (1991) na cultura do trigo, sob diferentes condiçōes microclimáticas. SI-D: sem irrigação e menos denso; $\mathrm{CI}-\mathrm{D}=\mathrm{com}$ irrigação e menos denso; $\mathrm{CI}+\mathrm{D}=\mathrm{com}$ irrigação e mais denso; $\mathrm{SI}+\mathrm{D}=$ sem irrigação e mais denso.

\section{REFERÊNCIAS BIBLIOGRÁFICAS}

ALFONSI, R.R.; PEDRO JÚNIOR, M.J.; ARRUDA, F.B.; ORTOLANI, A.A.; CAMARGO, M.B.P. de \& BRUNI$\mathrm{NI}, \mathrm{O}$. Métodos agrometeorológicos para controle da irrigação. Campinas, Instituto Agronômico, 1990. 62p. (Boletim tecnico, 133)

AMADOR, P.A. Duração do periodo de molhamento por orvalho: estimativa baseada em parâmetros meteorológicos e comparação do desempenho de instrumentos de medida. Piracicaba, 1987. 69p. Dissertação (Mestrado) - ESALQ-USP, 1987.

BARROS, B. de C. Avaliação da resistência varietal e da resposta de cultivares de trigo (Triticum aestivum $L$.) ao controle quimico de oidio, mancha marrom e ferrugem da folha. Piracicaba, 1988. 122p. Tese (Doutorado) - ESALQ-USP, 1988.
BLAD, B.L.; STEADMAN, J.R. \& WEISS, A. Canopy structure and irrigation influence white mold disease and microclimate of dry edible beans. Phytopathology, St. Paul, 68:1431-1437, 1978.

CAMPBELL, C.L. \& MADDEN, L.V. Introduction to plant disease epidemiology. New York, John Wiley \& Sons, 1990. 532p.

FELÍCIO, J.C.; CAMARGO, C.E. de O.; CAMARGO, M.B.P. de; CASTRO, J.L. de \& BARROS, B. de C. Trigo: três épocas de semeadura em Capão Bonito, SP, no período 1981-85. Bragantia, Campinas, 47(2):255-275, 1988.

FELÍCIO, J.C.; CAMARGO, C.E. de O.; FREITAS, J.G. de; FERREIRA FILHO, A.W.P.; BARROS, B. de C. \& CAMARGO, M.B.P. de. Avaliação de genótipos de trigo para a região do Vale do Paranapanema no quadriênio 1981-84. Bragantia, Campinas, 45(2):257$277,1986$. 
JAMES, W.C. An illustrated series of assessment keys for plant diseases, their preparation and usage. Canadian Plant Disease Survey, Ottawa, 51(2):39-65, 1971.

MENZIES, J.D. Plant diseases related to irrigation. In: HAGAN, R.M.; HAIZE, H.R. \& EDMINSTER, T.W., eds. Irrigation of agricultural lands. Madison, American Society of Agronomy, 1967. p.1058-1064. (Agronomy series, 11)

PEDRO JÚNIOR, M.J. Aspectos microclimáticos e epidemiologia. In: CURSO PRÁTICO INTERNACIONAL DE AGROMETEOROLOGIA PARA OTIMIZAÇÃO DA IRRIGAÇÃO, 3., Campinas, 1989. Campinas, Instituto Agronômico, 1989. 13p.

ROTEM, J. \& PALTI, J. Irrigation and plant disease. Annual Review of Phytopathology, St. Paul, 7:267$-288,1969$.
ROTEM, J.; PALTI, J. \& LOMAS, J. Effects of sprinkler irrigation at various times of the day on development of potato late blight. Phytopathology, St. Paul, 60:839-843, 1970.

SENTELHAS, P.C. Ocorrência de helmintosporiose (Helminthosporium sativum) $e$ de otdio (Erysiphe graminis) na cultura do trigo (Triticum aestivum, $L$.) sob diferentes condiçaes microclimáticas. Piracicaba, 1992. 103p. Dissertação (Mestrado) - ESALQ-USP, 1992.

SNEDECOR, G.W. \& COCHRAN, W.G. Statistical methods. 6.ed. Ames, Iowa State University Press, 1973. $593 \mathrm{p}$.

SOKAL, R.R. \& ROHLF, F.J. Biometry. San Francisco, W.H. Freeman, 1969. 776p.

SUTTON, J.C.; GILLESPIE, T.J. \& HILDEBRAND, P.D. Monitoring weather factors in relation to plant disease. Plant Disease, St. Paul, 68(1):78-84, 1984. 